\title{
Application of multiple regression for sensitivity analysis of helium line emissions to the electron density and temperature in Magnum-PSI
}

\author{
Shin Kajita, ${ }^{1, a)}$ Daisuke Nishijima, ${ }^{2}$ Keisuke Fujii, ${ }^{3}$ Gijs Akkermans, ${ }^{4}$ and Hennie van \\ der Meiden ${ }^{4}$ \\ ${ }^{1)}$ Institute of Materials and Systems for Sustainability, Nagoya University, \\ Nagoya 464-8603, Japan \\ ${ }^{2)}$ Center for Energy Research, University of California, San Diego, 9500 Gilman Dr., \\ La Jolla, CA 92093-0417, USA \\ ${ }^{3)}$ Graduate School of Engineering, Kyoto University, Kyoto 615-8540, \\ Japan \\ 4) NWO Institute DIFFER, Dutch Institute for Fundamental Energy Research, \\ De Zaale 20, 5612 AJ Eindhoven, The Netherlands
}

(Dated: 7 April 2021)

Helium line intensities have been utilized to measure the electron density, $n_{e}$, and temperature, $T_{e}$, by comparing measured line intensities to a collisional-radiative model (CRM). In this study, we use multiple regression analysis to train a model of the helium line intensities and $n_{e} / T_{e}$ obtained from a Thomson scattering system in the linear plasma device Magnum-PSI; based on the trained model, we predict $n_{e}$ and $T_{e}$ from line intensities. We show that this method can also obtain radial profiles of $n_{e}$ and $T_{e}$. We discuss appropriate selections of line pairs for the prediction based on the multiple regression analysis. A big advantage of this method against the standard technique using CRM is that modeling of atomic population distributions is not required, which sometimes needs to take into account various effects such as radiation trapping, transport of helium atoms in metastable states, etc.

\section{INTRODUCTION}

Line emissions from helium $(\mathrm{He})$ atoms have been utilized to measure the electron density, $n_{e}$, and temperature, $T_{e}$, in various fusion devices ${ }^{1-4}$. The basic principle of the measurement is to fit a relative population distribution obtained from a collisional radiative model $(\mathrm{CRM})$ to a measured one ${ }^{5}$. This method has also been used in various linear devices, and comparisons have been made to other diagnostics such as an electrostatic probe and laser Thomson scattering (TS $)^{6-11}$.

For the optimization process of CRM calculations, in addition to the dependence of the relative population distribution on $n_{e}$ and $T_{e}$, it is sometimes important to take into account several other effects including high energy electrons ${ }^{6}$, radiation trapping ${ }^{12}$, plasma fluctuations ${ }^{13,14}$, and transport of $\mathrm{He}$ atoms in metastable states ${ }^{14}$. However, it is not straightforward to model these effects inclusively. For instance, concerning the effect of radiation trapping, various investigations have been conducted in terms of neutral He density and temperature ${ }^{10,11}$, the radius and radial profile of the optical escape factor ${ }^{9,15}$. In particular, it is not easy to assess the influence far from the plasma column center in a linear plasma device, because the emissions from the central region significantly disturb the population distribution at the edge.

Recently, Nishijima and his colleagues have applied a machine learning method to the relations between He line intensities and $n_{e} / T_{e}$, and successfully reproduced radial profiles of $n_{e}$ and $T_{e}$ from optical emission spectroscopy (OES) data ${ }^{16}$. This method requires another

\footnotetext{
${ }^{a)}$ Electronic mail: kajita.shin@nagoya-u.jp
} 
reliable diagnostic tool, but does not require any sophisticated modeling of population distribution. Since $n_{e}$ was limited up to $\sim 4 \times 10^{18} \mathrm{~m}^{-3}$ in Ref. 16, it is of interest to check the validity of the method in a higher $n_{e}$ range, covering the divertor strike point region in fusion devices. Although a machine learning technique without a physics backbone cannot compete with a modeling that is able to treat all the relevant physics correctly, it can be a useful tool when the physics has yet to be fully understood. Also, the difference between machine learning and physics-based methods can give us clues to understand the physics further.

In this study, we analyze OES data collected at a higher $n_{e}$ range of $10^{19}-10^{21} \mathrm{~m}^{-3}$ in the Magnum-PSI linear device using multiple regression analysis to predict $n_{e}$ and $T_{e}$. Previously, in Magnum-PSI, it was found from the comparison between the OES data and CRM that $n_{e}$ and $T_{e}$ deduced from the OES were sometimes not consistent with those from $\mathrm{TS}^{11}$. In this study, in addition to a line of sight (LoS) observing the center of the plasma column, we will try to deal with radial profile data and discuss the robustness and limitation of the OES data. Moreover, based on the multiple regression analysis, selection of lines appropriate for the prediction of $n_{e}$ and $T_{e}$ is discussed.

\section{PREPARATION}

\section{A. Data set}

In this study, the data set in Ref. 11 that had 24 discharges at different discharge currents and gas pressures is used. The magnetic field strength was $1.2 \mathrm{~T}$. Details of the experimental device and experimental setup can be found in Refs. 11 and 17; here, a short explanation of the setup is provided. Pure He plasmas were produced in the linear plasma device Magnum-PSI. Figure 1 shows a schematic representing the field of views of the OES and TS seen from the target to the source. The second harmonic of Nd:YAG laser pulses $(532 \mathrm{~nm})$ pass through the plasma from the bottom to the top of the device ${ }^{18}$. The laser TS signal is collected from a side field of view and is detected by a high etendue transmission grating spectrometer that equips with an intensified charge coupled device. The Rayleigh peak, which is much narrower than TS, can be separated from the TS signals. The signal intensity is calibrated by Rayleigh scattering, enabling to measure $n_{e}$, while $T_{e}$ is evaluated from the spectrum broadening. The minimum measurable $n_{e}$ and $T_{e}$ are $1 \times 10^{17} \mathrm{~m}^{-3}$ and $0.07 \mathrm{eV}$, respectively. The radial profiles of $n_{e}$ and $T_{e}$ can be measured along the laser path. In this study, we assume the Thomson scattering system gives unbiased $n_{e}$ and $T_{e}$ data.

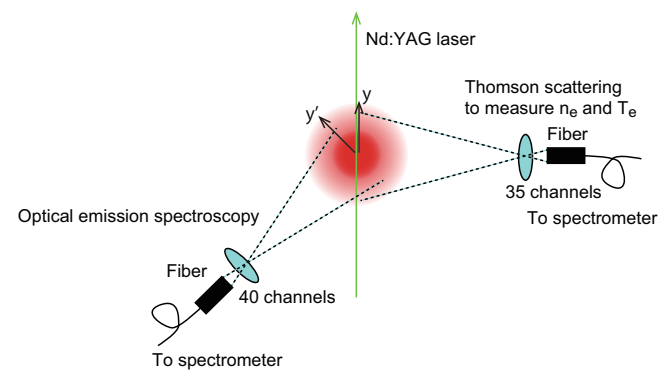

FIG. 1. A schematic representing the field of views of the OES and TS seen from the target to the source. Although the coordinates are different between TS $(y)$ and OES $\left(y^{\prime}\right)$, we will treat the data under the assumption that the parameter variation in the azimuthal direction was not significant for simplicity.

Figure 2 shows a typical observed emission spectrum. The wavelength coverage of the spectrometer used in this study is $\sim 165 \mathrm{~nm}$ in a single acquisition. At each plasma condition, two spectra were taken at two wavelength ranges: $\sim 365-530 \mathrm{~nm}$ and $\sim 660-825 \mathrm{~nm}$. Note 

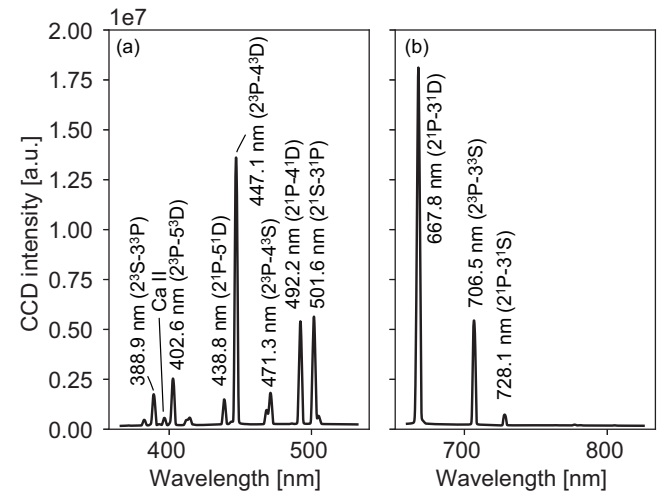

FIG. 2. A typical emission spectrum from the Magnum-PSI device used in this study.

TABLE I. The nine line intensities used in this study. The wavelengths and upper/lower states for the transitions are shown.

\begin{tabular}{|c|c|c|c|}
\hline Index & Wavelength $[\mathrm{nm}]$ & Upper state & Lower state \\
\hline 1 & $728.1 \mathrm{~nm}$ & $3^{1} \mathrm{~S}$ & $2^{1} \mathrm{P}$ \\
\hline 2 & $706.5 \mathrm{~nm}$ & $3^{3} \mathrm{~S}$ & $2^{3} \mathrm{P}$ \\
\hline 3 & $501.6 \mathrm{~nm}$ & $3^{1} \mathrm{P}$ & $2^{1} \mathrm{~S}$ \\
\hline 4 & $388.9 \mathrm{~nm}$ & $3^{3} \mathrm{P}$ & $2^{3} \mathrm{~S}$ \\
\hline 5 & $667.8 \mathrm{~nm}$ & $3^{1} \mathrm{D}$ & $2^{1} \mathrm{P}$ \\
\hline 6 & $492.2 \mathrm{~nm}$ & $4^{1} \mathrm{D}$ & $2^{1} \mathrm{P}$ \\
\hline 7 & $447.1 \mathrm{~nm}$ & $4^{3} \mathrm{D}$ & $2^{3} \mathrm{P}$ \\
\hline 8 & $438.8 \mathrm{~nm}$ & $5^{1} \mathrm{D}$ & $2^{1} \mathrm{P}$ \\
\hline 9 & $402.6 \mathrm{~nm}$ & $5^{3} \mathrm{D}$ & $2^{3} \mathrm{P}$ \\
\hline
\end{tabular}

that the strongest visible line at $587.6 \mathrm{~nm}$ was not measured in this study, as it often saturates when it is measured with other lines. And the line emission at $471.3 \mathrm{~nm}$ was not used, because there was an overlap with another line probably from some impurity. Other emission lines observed with a decent intensity are included in the analysis. Table I describes the wavelengths and transitions of the observed nine He atomic lines in the wavelength range of 388-728 nm. Those lines were observed by a Czerny-Turner type spectrometer. The intensities have been calibrated using a standard lamp; the calibration is, in principle, not necessary for the method used in this study. The axial (in the direction parallel to the magnetic field) position of the field of view for the OES was the same as the TS measurement, but it was rotated 135 degree in the clockwise direction from the LoS of the TS system, as shown in Fig. 1. The number of fiber channels was 40 for OES and 35 for TS. We will treat the data in the coordinate system shown in Fig. 1 with $y$ for TS and $y^{\prime}$ for OES under the assumption that the parameter variation in the azimuthal direction was not significant for simplicity.

Figure 3(a) shows the emission profile of the nine lines in a logarithmic scale, and Fig. $3(\mathrm{~b})$ shows the relative emission profiles normalized to the intensities at $y^{\prime}=0 \mathrm{~mm}$ in a linear scale. Figure 3(c) shows the radial profiles of $n_{e}$ and $T_{e}$ measured by TS. In this study, we split radial profiles to three regions: core $(r<3 \mathrm{~mm})$, transition region $(3<r<7 \mathrm{~mm})$, and periphery $(r>7 \mathrm{~mm})$, where $r$ is the distance from the center of the plasma column. The profile at $501.6 \mathrm{~nm}$ has a relatively strong intensity at the periphery similar to the other devices ${ }^{10}$. This is because the upper state of the transition at $501.6 \mathrm{~nm}$ is $3^{1} \mathrm{P}$, which is also associated with a resonance line at $53.7 \mathrm{~nm}\left(1^{1} \mathrm{~S}-3{ }^{1} \mathrm{P}\right)$, and the photoexcitation by the radiation from the center can increase the population at the periphery ${ }^{19,20}$.

Figure 4 shows the distributions of $T_{e}$ and $n_{e}$ that will be used for the analysis in this 


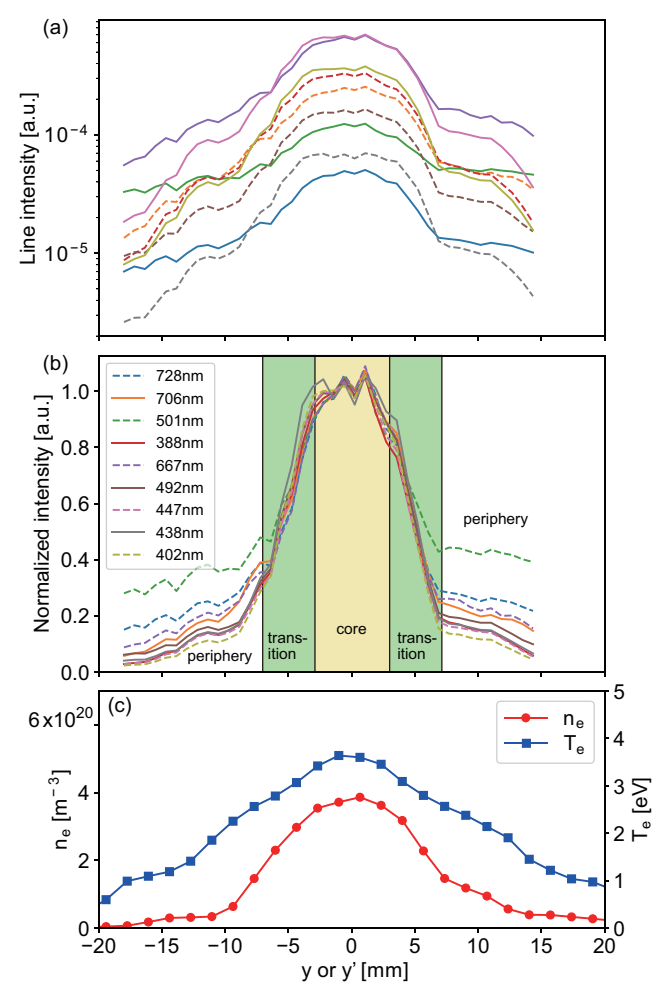

FIG. 3. (a) The emission profile of the nine lines in a logarithmic scale, (b) the relative emission profiles normalized to the intensities at $y^{\prime}=0 \mathrm{~mm}$ in a linear scale, and (c) the radial profiles of $n_{e}$ and $T_{e}$ measured by TS.

study; different markers represent the three radial regions (core, transition, and periphery). In the core and transition regions, $n_{e}$ and $T_{e}$ are mainly in the ranges of $10^{20}-10^{21} \mathrm{~m}^{-3}$ and $0.2-3 \mathrm{eV}$, respectively. In the periphery, the density is lower than the core and transition region, and $n_{e}$ and $T_{e}$ are mainly in the ranges of $10^{19}-10^{20} \mathrm{~m}^{-3}$ and $0.2-2 \mathrm{eV}$, respectively.

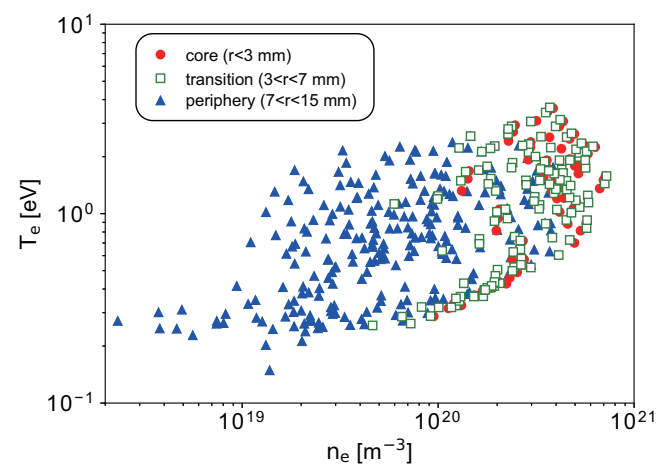

FIG. 4. The distributions of $T_{e}$ and $n_{e}$ that will used in this study from the three regions (core, transition, and periphery). 


\section{B. Methods}

In this study, we mainly use multiple regression analysis (LinearRegression in Scikitlearn ${ }^{21}$ ) and partially use a non-linear model, Kernel ridge regression (KernelRidge in Scikit-learn). The main reason for using a linear model is that we can interprete the coefficients in an easy manner.

In the regression analysis, the relations between $n_{e}$ or $T_{e}$ from TS and line intensities are trained. The TS data was interpolated to the corresponding OES radial position. Because only the relative population distribution is important, the intensities are normalized to the sum of the used ones as

$$
I_{i}=\frac{\iota_{i}}{\sum_{j} \iota_{j}},
$$

where $\iota_{i}$ is the line intensity with the index number of $i$, which is shown in table I. Then, we take the logarithm for all the above values, and they are used for the input values of the model. In other words, in the multiple regression analysis, we assume that $n_{e}$ and $T_{e}$ can be expressed in the following form:

$$
n_{e} \text { or } T_{e}=C_{0} I_{1}^{C_{1}} I_{2}^{C_{2}} \cdots I_{n}^{C_{n}},
$$

where $C_{0}, C_{1}, C_{2}, \cdots$, and $C_{N}$ are coefficients. When taking logarithm on both sides, Eq. (2) becomes

$$
\log n_{e} \text { or } \log T_{e}=\log C_{0}+C_{1} \log I_{1}+C_{2} \log I_{2}+\cdots+C_{n} \log I_{n} .
$$

Although there is no theoretical support that ne and Te can be expressed using Eq. (2), Nishijima et al. ${ }^{16}$ have recently shown that the power function model using the relation in Eq. (2) can be well used for the prediction of $n_{e}$ and $T_{e}$ in the ionizing plasmas in the PISCES-A linear device. Thus, in this study, we mainly use the linear model, and the deviation from Eq. (2) is discussed using a non-linear model. First, in Sec. III, among the available 24 discharge data, $2 / 3$ (16 discharges) of them are selected randomly and used for training, and remained $1 / 3$ (8 discharges) is used for testing.

\section{MULTIPLE REGRESSION ANALYSIS}

First, we use all the radial profile data at once for training and testing. Figure 5(a,b) shows predicted $n_{e}$ and $T_{e}$, respectively, as a function of the measured TS values. As mentioned in Sec. II B, randomly selected two-thirds of available discharges were used for training and remaining discharges were used for testing. We repeated the random selection three times and plotted in Fig. 5(a,b) with different markers. The scattering of predicted $n_{e}$ is not small and has a range from $10^{19}-10^{20} \mathrm{~m}^{-3}$ at TS-measured $n_{e} \approx 2 \times 10^{19} \mathrm{~m}^{-3}$. The scattering of the predicted $T_{e}$ also has a wide range, and the maximum scattering is roughly a factor of five.

To specify the origin of the scattering, the data set was separated to the three regions (core, transition, and periphery), and training and testing were performed in the three regions separately. Figure $6(\mathrm{a}, \mathrm{b})$ plots the predicted $n_{e}$ and $T_{e}$, respectively, as a function of measured TS values for the three regions (core: circles, transition: triangles, and periphery: crosses). The blue and red colors represent the different sets of data used for training and testing. It is seen that the residual errors are relatively small in the core region, while they gradually increase with increasing radius.

In Table II, the multiple correlation coefficient, $R$, and the residual errors, $e$, are presented. In this study, $e$ is defined as

$$
e=\frac{1}{n} \sum_{j} \frac{\left|X_{j}-x_{j}\right|}{X_{j}}
$$



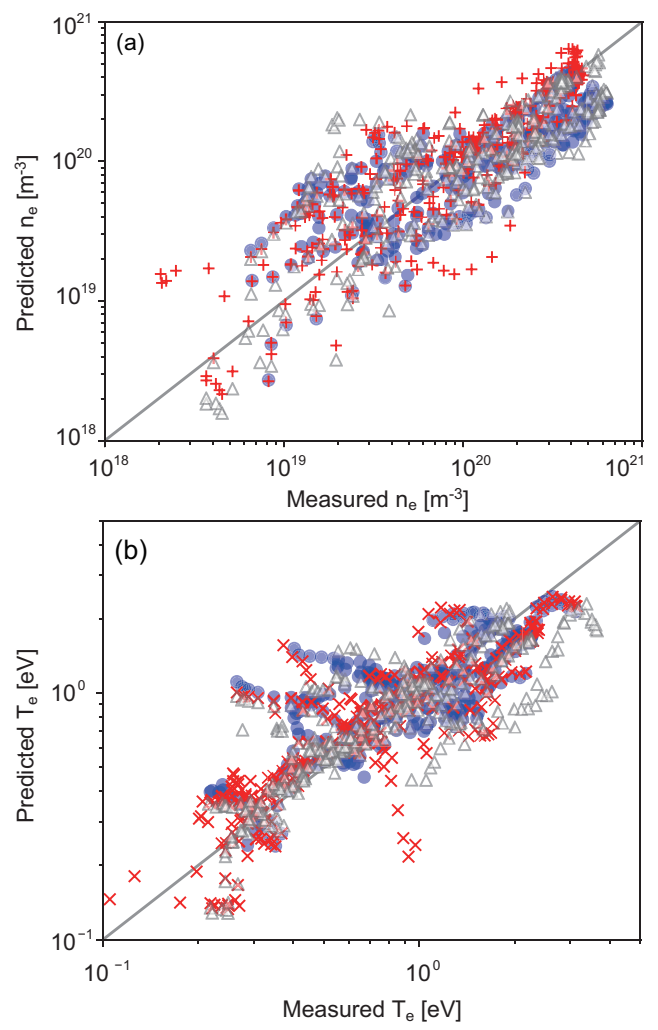

FIG. 5. Predicted (a) $n_{e}$ and (b) $T_{e}$ of test data as a function of TS-measured $n_{e}$ and $T_{e}$, respectively. All the radial profile data were analyzed together. Different markers represent the results using randomly chosen three different set of data for training/testing.

TABLE II. The multiple correlation coefficient, $R$, and the residual errors assessed from the multiple regression analysis in Figs. 5 and 6 . Here, All in the region of interest means that all the three regions were used for the analysis together, and Core, Transition, and Periphery mean the three regions were used for the analysis separately.

\begin{tabular}{ccc} 
Region of interest & $R$ coefficient & Residual error $(\%)$ \\
\hline \hline All $\left(n_{e}\right)$ & $0.81 \pm 0.05$ & 80.3 \\
All $\left(T_{e}\right)$ & $0.75 \pm 0.11$ & 37.9 \\
Core $\left(n_{e}\right)$ & $0.82 \pm 0.11$ & 19.8 \\
Core $\left(T_{e}\right)$ & $0.89 \pm 0.07$ & 17.0 \\
Transition $\left(n_{e}\right)$ & $0.79 \pm 0.08$ & 29.1 \\
Transition $\left(T_{e}\right)$ & $0.74 \pm 0.15$ & 25.1 \\
Periphery $\left(n_{e}\right)$ & $0.55 \pm 0.13$ & 90.7 \\
Periphery $\left(T_{e}\right)$ & $0.45 \pm 0.19$ & 45.5 \\
\hline \hline
\end{tabular}

where $X_{j}$ and $x_{j}$ are measured and predicted values, respectively, $j$ is the index of the data. We repeated random selections of the test/train data 100 times, and the summation was taken for all the test data. Even with including all the radial data at once (Fig. 5), $R$ is 0.81 for $n_{e}$ and is 0.75 for $T_{e}$, indicating that the correlation is not so bad. However, $e$ for $n_{e}$ is $80 \%$, which is not sufficiently low. When focusing on the core region, both $R$ and $e$ are improved. In particular, $e$ is reduced to less than $20 \%$ for both $n_{e}$ and $T_{e}$. As shifting to the outer regions, $R$ gradually decreases and $e$ increases. Thus, the errors are found to originate mainly from the periphery in the current data set. 

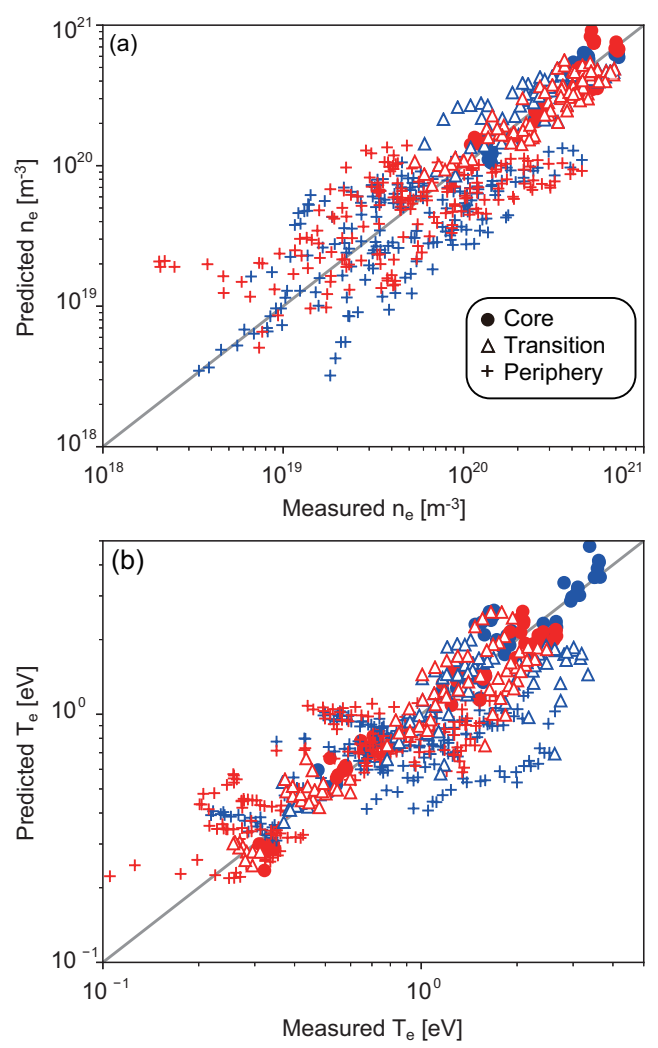

FIG. 6. Predicted (a) $n_{e}$ and (b) $T_{e}$ of the test data as a function of TS-measured $n_{e}$ and $T_{e}$, respectively. The training and testing were performed in the three regions separately. Different markers represent the different regions (core: circles, transition: triangles, and periphery: crosses), and blue and red colors correspond to randomly chosen two different sets of data for training/testing.
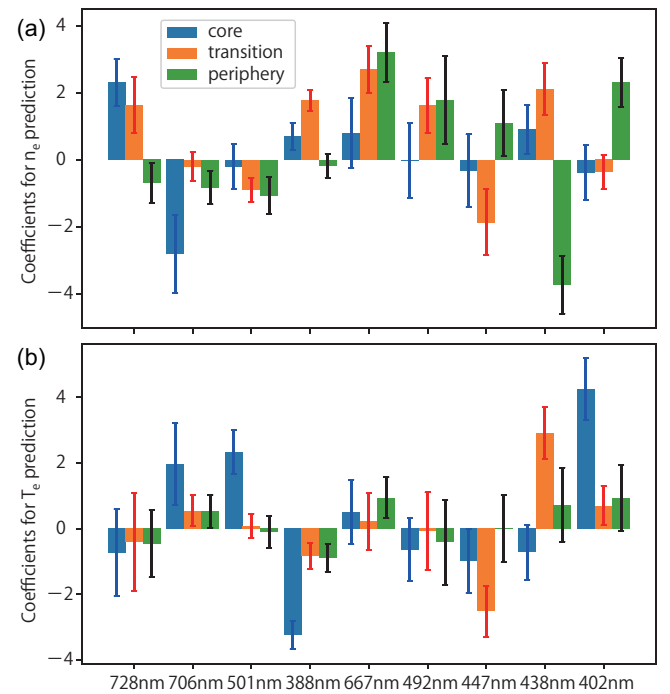

FIG. 7. Obtained coefficients of the nine lines for (a) $n_{e}$ and (b) $T_{e}$ predictions at the three different regions. We repeated the analysis 100 times and the average and standard deviation of the values were plotted as bars and error bars, respectively. 
Figure $7(\mathrm{a}, \mathrm{b})$ shows the obtained coefficients of the nine lines for $n_{e}$ and $T_{e}$ predictions, respectively, at the three different regions. Here, to improve statistics from Fig. 6, where calculations were done only twice, we repeated the analysis with random selection of test/train data 100 times, and the average and standard deviation values are plotted as bars with error bars. It is found that the coefficient values are different in the three different regions. For example, the coefficients of 728.1 and $438.8 \mathrm{~nm}$ for the $n_{e}$ prediction alter from positive to negative values as shifting from the core to the periphery. One of the potential causes is the effect of radiation transport. Previously, it was revealed from both experiments in the PISCES-A device and a ray tracing simulation that the intensity of singlet lines is enhanced especially at the periphery of the plasma column, because of the absorption of resonance lines emitted from the brighter plasma central region, while the enhancement of the triplet line intensity is much smaller over the whole profile ${ }^{19}$. For example, the population densities in $3{ }^{1} \mathrm{P}$ and $3{ }^{1} \mathrm{~S}$ states increased by three orders and an order of magnitude, respectively, due to the absorption of resonance lines. This means that the absorption effect is more dominant than electron-impact excitation processes to populate the singlet states. As shown in Fig. 3(b), the $501.6 \mathrm{~nm}$ and $728.1 \mathrm{~nm}$ lines have relatively higher intensities at the periphery, because the upper states of these line are $3^{1} \mathrm{P}$ and $3^{1} \mathrm{~S}$, respectively. Thus, these lines are more sensitive to the absorption effect and are less sensitive to electron-impact excitation, i.e. $n_{e}$ and $T_{e}$, especially at the periphery. On the other hand, lines that are less sensitive to absorption are thought to be more beneficial for the prediction of $n_{e}$ and $T_{e}$ at the periphery. In addition to the value itself, the relation between the value and the scattering (error bar) is also important. If the scattering is comparable or larger than the value itself, it is suggested that the value is not stable and does not have a clear sensitivity to the parameter. Concerning the coefficient of, e.g., $728.1 \mathrm{~nm}$ for $T_{e}$ prediction, the error bars are larger than the coefficient values, suggesting that the line intensity is not sensitive to the $T_{e}$ prediction. We will use Fig. $7(\mathrm{a}, \mathrm{b})$ later for practical selection of lines.

Although the error is large at the periphery region, the fact that $R$ is $\sim 0.5$ suggests the predicted values have correlation with the measured values to some extent. Here we try to reconstruct the radial $n_{e}$ and $T_{e}$ profiles from the OES data. Figure $8(\mathrm{a}, \mathrm{b})$ shows those from OES and TS. Here, one discharge was selected for test data and all the other data were used for training. For assessing the errors of $n_{e}$ and $T_{e}$, we repeated the analysis $(1 / 3$ test data and 2/3 training data) used in Fig. 6(a,b) 100 times, and standard deviations of the TS $n_{e}$ and $T_{e}$ of the test data that have close predicted $n_{e}$ and $T_{e}(< \pm 10 \%)$ values, respectively, were used. While the errors are relatively large, the predicted radial profiles of $n_{e}$ and $T_{e}$ from OES agree well with those of TS. It should be noted that OES coupled with CRM was not able to reproduce the radial profiles from $\mathrm{TS}^{11}$.

\section{SELECTION OF LINES}

In Sec. III, nine lines were used for the analysis as in the previous study ${ }^{11}$, where the quality of the $n_{e}$ and $T_{e}$ predictions using CRM was deteriorated when reducing the number of lines. In this section, we assess the importance of each line and discuss the best selections of necessary lines.

To select lines, let us go back to Fig. 7. For the first step, we focus on the core region in this study. We can eliminate lines that have little sensitivity to both $n_{e}$ and $T_{e}$. It is apparent that lines at 728.1 and $706.5 \mathrm{~nm}$ have sensitivity to $n_{e}$, and lines at $706.5,501.6$, 388.9 , and $402.6 \mathrm{~nm}$ have sensitivity to $T_{e}$, while lines at $667.8,492.2,447.1,438.8 \mathrm{~nm}$ seem not to be so sensitive to both $n_{e}$ and $T_{e}$. We assess the performance for six cases (i)-(vi) with different line selections shown in Table III. Case (i) uses all the nine lines, case (ii) eliminates two lines $(667.8$ and $492.2 \mathrm{~nm}$ ) from case (i), case (iii) eliminates two more lines (447.1 and $438.8 \mathrm{~nm}$ ) from case (ii), and cases (iv)-(v) are the cases used previously ${ }^{14}$. The three lines used in case (vi) are the popular lines that have been used frequently ${ }^{5-10}$. In case $(\mathrm{v})$, the line at $501.6 \mathrm{~nm}$, which is sensitive to radiation trapping ${ }^{20}$, is added to 

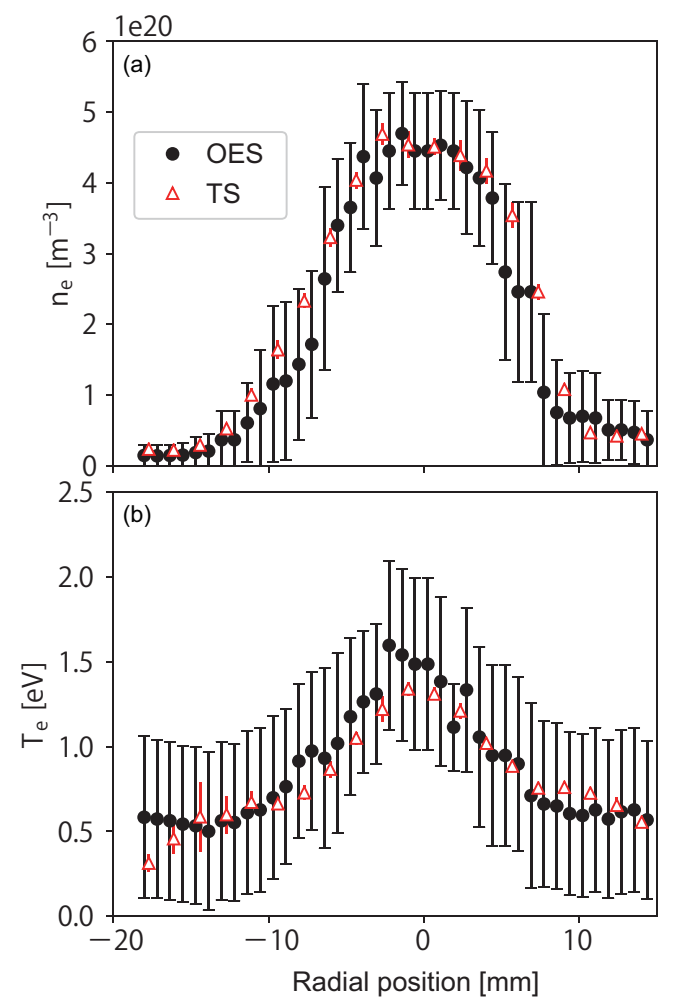

FIG. 8. Predicted radial profiles of (a) $n_{e}$ and (b) $T_{e}$ from OES (closed circles) compared with measured TS values (open triangles).

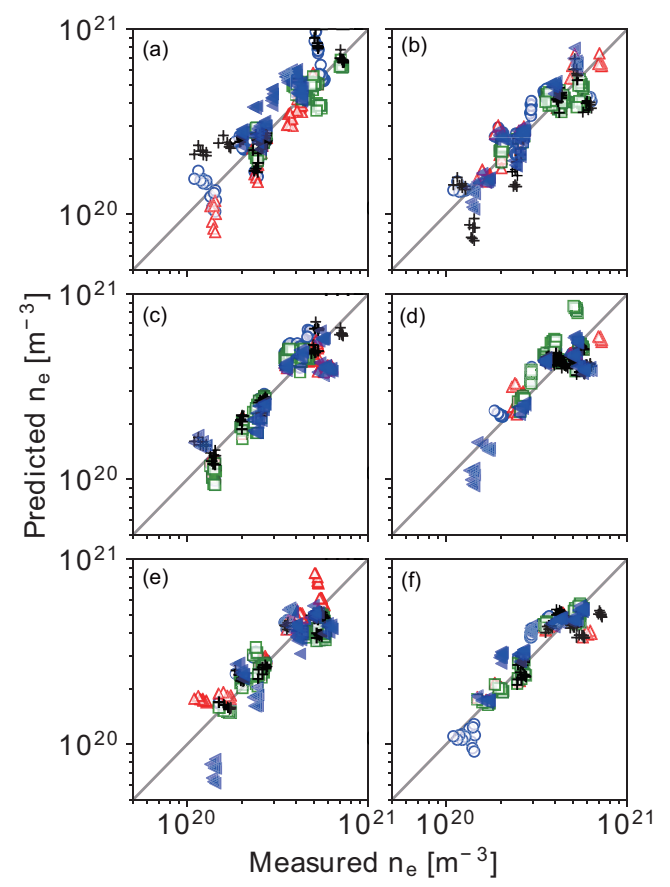

FIG. 9. (a-f) Predicted $n_{e}$ in the core region as a function of the measured $n_{e}$ for different line selection cases (i)-(vi), respectively. Different markers represent randomly chosen five different set of data for training/testing. 
TABLE III. Six cases (i)-(vi) with different lines used for the analysis.

\begin{tabular}{c|c|l} 
Case & Number of lines & Wavelengths (nm) \\
\hline \hline (i) & 9 & $728.1,706.5,501.6,388.9,667.8,492.2,447.1,438.8,402.6$ \\
(ii) & 7 & $728.1,706.5,501.6,388.9,447.1,438.8,402.6$ \\
(iii) & 5 & $728.1,706.5,501.6,388.9,402.6$ \\
(iv) & 5 & $728.1,706.5,501.6,667.8,447.1$ \\
(v) & 4 & $728.1,706.5,501.6,667.8$ \\
(vi) & 3 & $728.1,706.5,667.8$ \\
\hline
\end{tabular}

case (vi). In case (iv), another line at $447.1 \mathrm{~nm}$, which is sensitive to the recombining component $^{14}$, is added to case (v).

Figure 9 (a-f) plots the predicted $n_{e}$ in the core region as a function of the TS-measured $n_{e}$ for cases (i)-(vi), respectively. Again, randomly chosen 2/3 (16 discharges) data from the data set was used for training and the remained 1/3 (8 discharges) data was used for testing. Different markers in Fig. 9 represent five sets of training and testing. It is seen that the predicted values are almost consistent with TS values, suggesting that the method can be used for all the cases. It is interesting to note that the quality of the prediction is good even with three lines for $n_{e}$, i.e. case (vi). Figure 10 (a-f) plots the predicted $T_{e}$ in the core region as a function of the measured $T_{e}$ for cases (i)-(vi), respectively. While the results are almost consistent for cases (i)-(iii), the quality of the prediction is worth in cases (iv)-(vi), especially, at $T_{e}>2 \mathrm{eV}$.
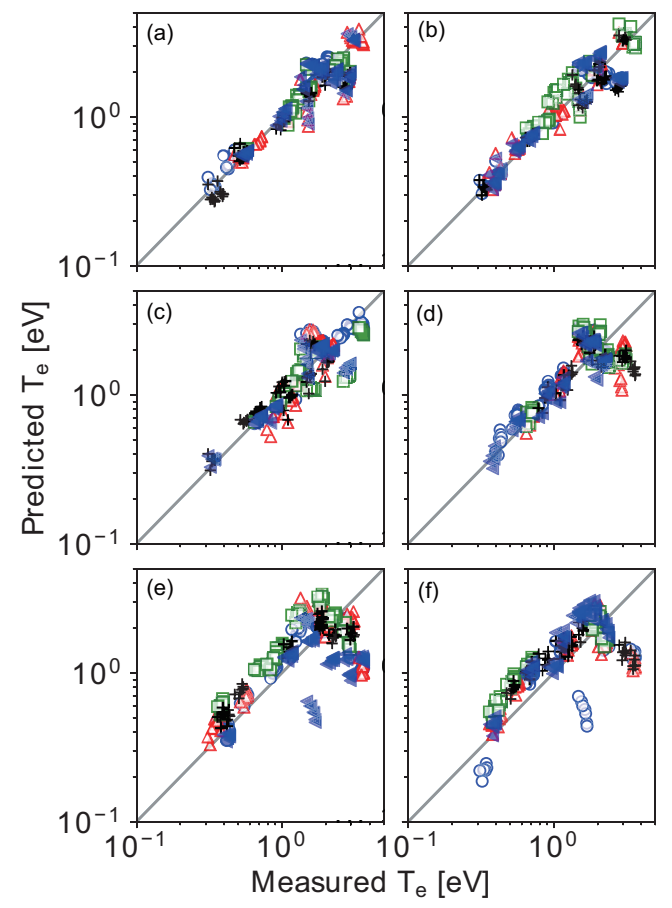

FIG. 10. (a-f) Predicted $T_{e}$ in the core region as a function of the TS-measured $T_{e}$ for different line selection cases (i)-(vi), respectively. Different markers represent randomly chosen five different set of data for training/testing.

Figure 11 summarizes the residual errors in $n_{e}$ and $T_{e}$ for the different line selection cases (i)-(iv). The error of $n_{e}$ slightly decreases when the number of lines decreases from nine to three, but it does not depend strongly on the selection of the lines and is in a range of 


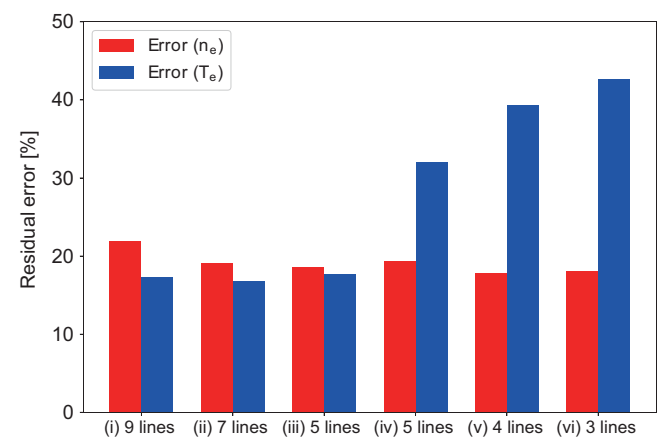

FIG. 11. The residual errors in $n_{e}$ and $T_{e}$ for cases (i)-(vi).

$17-22 \%$. On the other hand, the $T_{e}$ error is nearly constant at $\sim 15 \%$ for cases (i)-(iii); it increases to $\approx 30 \%$ in case (iv) and further increased to $\approx 40 \%$ in cases (v) and (vi). This might be caused by the usage of the linear model for training the data. Thus, we examined a non-linear fit model (Kernel ridge regression) for cases (iv)-(vi). As shown in Fig. 12, the predicted $T_{e}$ using the Kernel ridge regression still deviates from the TS-measured values, in particular, at $T_{e}>2 \mathrm{eV}$, as with the linear model. The residual errors of cases (iv)-(vi) are $28.1,37.2$, and $40.9 \%$, respectively. The error was slightly improved for case (iv), but almost no improvement for cases (v) and (vi).

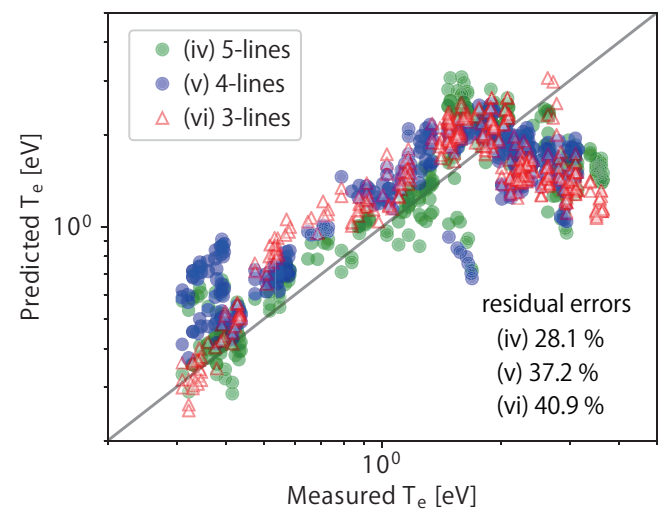

FIG. 12. Predicted $T_{e}$ using the Kernel ridge regression plotted as a function of the TS-measured $T_{e}$ for cases (iv)-(vi).

Fig. 13(a) plots the line intensity ratios of $728.1 / 706.5 \mathrm{~nm}$ vs. $667.8 / 728.1 \mathrm{~nm}$ with $T_{e}$ as the color of markers. Here, only the core region data are used. The line intensity ratios $728.1 / 706.5 \mathrm{~nm}$ and $667.8 / 728.1 \mathrm{~nm}$ are sensitive mainly to $T_{e}$ and $n_{e}$, respectively. However, high and low $T_{e}$ data points overlap at 728.1/706.5 $\mathrm{nm} \sim 0.06$ and 667.8/728.1 $\mathrm{nm} \sim 24$, indicating that $T_{e}$ is a multivalued function of this line intensity ratio pair. This may be explained by the mixture of ionizing and recombining components ${ }^{9}$, since there is a minimum of the $728.1 / 706.5 \mathrm{~nm}$ ratio around the boundary between ionizing (higher $T_{e}$ ) and recombining (lower $T_{e}$ ) plasmas. While the addition of $501.6 \mathrm{~nm}$ to the three lines (728.1, 706.5, and $667.8 \mathrm{~nm}$ ) does not reduce the deviation at $T_{e}>2 \mathrm{eV}$ [see Fig. 10 (e) and (f)], the fit is improved by the further addition of $447.1 \mathrm{~nm}$ to the above four lines [see Fig. 10 (d)]. In Fig. 13(b), a ratio 402.6/501.6 nm is plotted against 501.6/388.9 nm with $T_{e}$ as the color of markers. Here, the two ratios consist of the three most sensitive lines to $T_{e}$ in the core, as shown in Fig. 7 . The lower $T_{e}$ region exists at the bottom and the higher $T_{e}$ region exists at the upper right of the figure. In Fig. 13(b), the mixture of different $T_{e}$ regions is less than that in Fig. 13(a). Thus, as is suggested in Fig. 10(a-c), the line 
selection of cases (i)-(iii) is more powerful to separate the contributions from ionizing and recombining components compared to case (iv)-(vi).

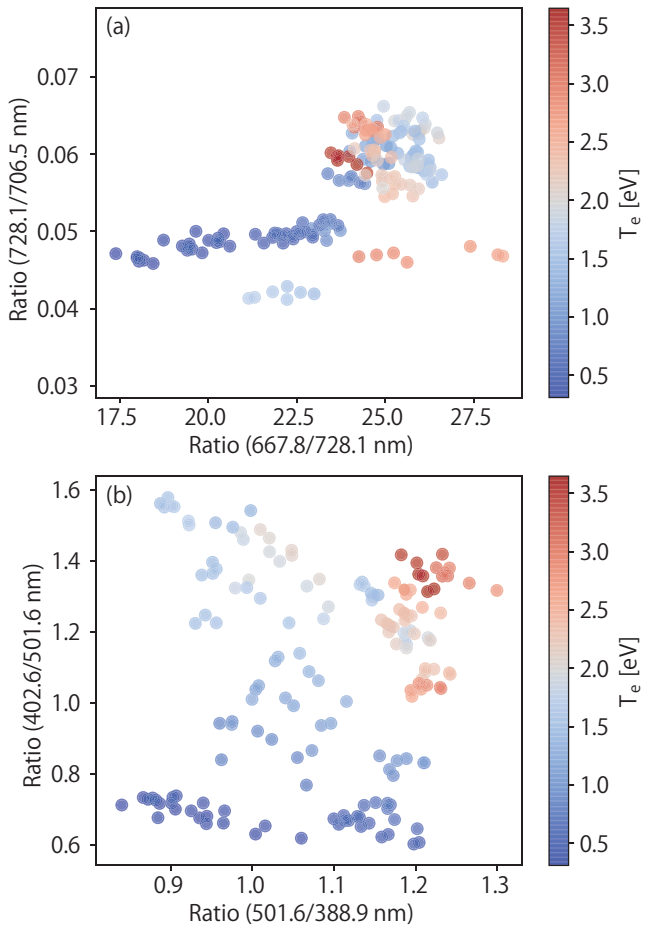

FIG. 13. Distributions of the line intensity ratios in the core region: (a) $728.1 / 706.5 \mathrm{~nm}$ vs. $667.8 / 728.1 \mathrm{~nm}$ and (b) 402.6/501.6 nm vs. 501.6/388.9 nm under various discharge conditions. The color of the marker represents TS-measured $T_{e}$ values. This kind of plot is useful to find out if any overlaps of different $T_{e}$ values exist in a line ratio pair.

In this section, we discussed the line selection, focusing on the data in the core region. However, as expected from Fig. 7, the best selection of lines can be different in the transition and periphery regions. Future work will focus on the development of robust tools for prediction that can be applied to various regions and other devices.

\section{CONCLUSIONS}

We showed that the machine learning methods can be used to predict the electron density $\left(n_{e}\right)$ and temperature $\left(T_{e}\right)$ from helium $(\mathrm{He})$ line intensities if there is another reliable measurement method for $n_{e}$ and $T_{e}$ that can be used for training. Multiple regression analysis was applied to train the data set of optical emission spectroscopy (OES) data and $n_{e} / T_{e}$ from Thomson scattering (TS) in the linear plasma device Magnum-PSI. The intensity of nine He I lines was used for the analysis: 728.1, 706.5, 501.6, 388.9, 667.8, 492.2, 447.1, 438.8, and $402.6 \mathrm{~nm}$.

When using all the radial data set at once, the residual errors were large: $\approx 80 \%$ for $n_{e}$ and $\approx 40 \%$ for $T_{e}$. Thus, the radial profile was separated into the core $(r<3 \mathrm{~mm})$, transition $(3<r<7 \mathrm{~mm})$, and periphery $(r>7 \mathrm{~mm})$ regions to identify the region, the data of which caused the large residual errors. It was found that the data at the periphery caused the large residual errors, probably because the population densities of, mainly, singlet states at the periphery are altered by absorption of photons transported from the core to the periphery. Based on the coefficients derived from the multiple regression analysis of the data in the core region, it was found that a satisfactory prediction of $n_{e}$ and $T_{e}$ requires, at least, the 
following five lines at 728.1, 706.5, 501.6, 388.9, and $402.6 \mathrm{~nm}$. With this line selection, the error, originating from the mixture of ionizing and recombining components, is reduced.

This study demonstrated that machine learning can be a powerful tool for OES measurement to predict $n_{e}$ and $T_{e}$ in case there is another diagnostic for training a model. This method has an advantage, which does not require any complicated modeling for population distribution of He atoms, e.g. the effects of radiation trapping, neutral density/temperature, transport of metastable state atoms. In this study, we focused on the data from one device, which covers the $n_{e}$ and $T_{e}$ ranges of $10^{19}-10^{21} \mathrm{~m}^{-3}$ and $0.2-3 \mathrm{eV}$, respectively. In this study, we used the laser Thomson scattering system, which is reliable with small measurement errors, as an independent diagnostic to train the model. Even if the accuracy of an independent measurement system is low, we can make a regression analysis with enough training data unless those are biased. The trained model is expected to predict $n_{e}$ and $T_{e}$ with a better accuracy than the independent measurement system, since the line intensity is usually observed with a good signal to noise ratio. In the future, it is of interest to investigate the property of OES data from various experimental devices, including other linear devices as well as tokamak and helical fusion devices.

\section{ACKNOWLEDGEMENT}

This work was supported in part by a Grant-in-Aid for Scientific Research 19H01874 and Fund for the Promotion of Joint International Research 17KK0132 from the Japan Society for the Promotion of Science (JSPS).

${ }^{1}$ M. Griener, E. Wolfrum, M. Cavedon, R. Dux, V. Rohde, M. Sochor, J. M. Munoz Burgos, O. Schmitz and U. Stroth: Review of Scientific Instruments 89 (2018) 10 D102.

${ }^{2}$ M. Goto and K. Sawada: Journal of Quantitative Spectroscopy and Radiative Transfer 137 (2014) 23.

${ }^{3}$ M. Agostini, P. Scarin, R. Milazzo, V. Cervaro and R. Ghiraldelli: Review of Scientific Instruments 91 (2020) 113503.

${ }^{4}$ S. Ma, J. Howard, B. D. Blackwell and N. Thapar: Review of Scientific Instruments 83 (2012) 033102.

${ }^{5}$ M. Goto: J. Qunantitative Spectroscopy and Radiative Transfer 76 (2003) 331.

${ }^{6}$ S. Sasaki, S. Takamura, S. Watanabe, S. Masuzaki, T. Kato and K. Kadota: Rev. Sci. Instrum. 67 (1996) 3521.

${ }^{7}$ R. F. Boivin, J. L. Kline and E. E. Scime: Physics of Plasmas 8 (2001) 5303.

${ }^{8}$ Y. Iida, S. Kado, A. Okamoto, S. Kajita, T. Shikama, D. Yamasaki and S. Tanaka: J. Plasma Fusion Research SERIES 7 (2006) 123.

${ }^{9}$ S. Kajita, N. Ohno, S. Takamura and T. Nakano: Physics of Plasmas 13 (2006) 013301.

${ }^{10}$ D. Nishijima and E. M. Hollmann: Plasma Physics and Controlled Fusion 49 (2007) 791.

${ }^{11} \mathrm{~S}$. Kajita, G. Akkermans, K. Fujii, H. van der Meiden and M. C. M. van de Sanden: AIP Advances 10 (2020) 025225.

${ }^{12}$ T. Fujimoto, Plasma spectroscopy (Clarendon press, Oxford, 2004).

${ }^{13}$ F. B. Rosmej, N. Ohno, S. Takamura and S. Kajita: Contrib. Plasma Phys. 48 (2008) 243.

${ }^{14}$ S. Kajita, K. Suzuki, H. Tanaka and N. Ohno: Physics of Plasmas 25 (2018) 063303.

${ }^{15}$ Y. Iida, S. Kado and S. Tanaka: Physics of Plasmas 17 (2010) 123301.

${ }^{16}$ D. Nishijima, S. Kajita and G. R. Tynan: Review of Scientific Instruments 92 (2021) 023505.

${ }^{17}$ J. Rapp et al.: Fusion Engineering and Design 85 (2010) 1455 .

${ }^{18}$ H. J. van der Meiden et al.: Review of Scientific Instruments 83 (2012) 123505.

${ }^{19}$ S. Kajita, D. Nishijima, E. M. Hollmann and N. Ohno: Physics of Plasmas 16 (2009) 063303.

${ }^{20}$ S. Kajita and N. Ohno: Rev. Sci. Instrum. 82 (2011) 023501.

${ }^{21} \mathrm{~F}$. Pedregosa et al:: the Journal of machine Learning research 12 (2011) 2825. 